\title{
A REGENERAÇÃO DE UMA FLORESTA TROPICAL MONTANA APÓS CORTE E QUEIMA (SÃO PAULO-BRASIL)
}

\author{
TABARELLI, M. ${ }^{1}$ e MANTOVANI, W. ${ }^{2}$ \\ 'Departamento de Botânica, CCB, Universidade Federal de Pernambuco, \\ Rua Moraes Rego, s/n, CEP 50670-901, Recife, PE \\ ${ }^{2}$ Departamento de Ecologia Geral, Universidade de São Paulo, C.P. 11461, CEP 05422-970, São Paulo, SP \\ Correspondência para: Marcelo Tabarelli, Departamento de Botânica, CCB, Universidade Federal \\ de Pernambuco, Rua Moraes Rego, s/n, CEP 50670-901, Recife, PE \\ Recebido em 02/12/97 - Aceito em 17/06/98 - Distribuído em 30/06/99
}

(Com 3 figuras)

\section{ABSTRACT \\ Regeneration of a neotropical montane forest following slash-and-burn (São Paulo-Brazil)}

Slash-and-burn has been the main kind of disturbance in the Atlantic montane forest. We analyzed the change in vegetation structure, woody species composition, species richness and diversity and plant guild composition after the cutting and burning of a mature forest. According to our results the rate of vegetation change is related to the forest attribute analyzed. In the first moment, the disturbed forest had the species richness and diversity restored, followed by plant guild composition, species composition and finally its physical structure. A forty year old forest site showed between $38.8 \%$ and $117.1 \%$ of the values recorded in the mature site, suggesting that this forest regenerates following patterns observed in other tropical forests. In addition to disturbance intensity, this montane forest has particular features as the average size of diaspores and tree species richness, which are thought to provide this forest higher resilience. Changes in woody species composition can be described by successional models formed by three or four stages, depending on the disturbance intensity. Models are characterized by pioneer species of Imperata, Pteridium, Baccharis, Tibouchina and Alchornea genera.

Key words: Atlantic montane forest, forest regeneration, resilience, slash-and-burn.

\section{RESUMO}

O corte e queima são um dos tipos de perturbação predominante na floresta Atlântica montana no Sudeste do Brasil. A análise de uma cronossequiência de regeneração (10, 18, 40 anos e floresta madura) corrobora a hipótese de que as características da floresta se transformam em diferentes velocidades em direção aos valores observados na floresta madura. Em ordem decrescente de velocidade, a floresta, após corte e queima, restaura a riqueza e a diversidade de espécies arbóreo-arbustivas, a composição de guildas, a composição florística (importância relativa das famílias) e, por último, os atributos da estrutura física, com exceção da densidade de indivíduos. A floresta com 40 anos apresentou entre $38,8 \%$ e $117,1 \%$ dos valores observados na floresta madura, sugerindo que esta se regenera dentro do padrão observado para o conjunto das florestas tropicais. Além da intensidade de perturbação, determinadas características dessa floresta montana, como o tamanho médio de diásporos e a importância relativa de grupos dispersores, podem conferir-lhe maior resiliência. As transformações florísticas direcionais, observadas durante a regeneração, podem ser descritas através de modelos sucessionais compostos por quatro ou cinco fases, dependendo da intensidade de perturbação. Nesses modelos, as espécies dominantes pertencem aos gêneros Imperata, Pteridium, Baccharis, Tibouchina e Alchornea.

Palavras-chave: corte e queima, floresta Atlântica montana, regeneração, resiliência. 


\section{INTRODUÇÃO}

A regeneração da floresta é definida como o processo pelo qual a floresta perturbada atinge características da floresta madura (Klein, 1980; Saldarriaga \& Uhl, 1991), o que pressupõe modificações nas características da comunidade e mudanças direcionais na composição de espécies (Budowski, 1965; Klein, 1980; Gómez-Pompa \& Vásquez-Yanes, 1981; Whitmore, 1991; Kappelle et al., 1996).

Conforme Whitmore (1990) e Guariguata \& Dupuy (1997), a velocidade de regeneração da floresta tropical depende da intensidade da perturbação sofrida. O tempo de regeneração de algumas florestas tropicais americanas foi estimado entre 150 e 200 anos (Unesco/Pnuma/Fao, 1980). Saldarriaga \& Uhl (1991) estimaram que são necessários, aproximadamente, 140 a 200 anos para que a floresta tropical de terra firme, estabelecida em áreas de cultivo abandonadas, apresente valores de biomassa similares aos da floresta madura na Amazônia venezuelana.

Conforme Ewel (1980), as florestas tropicais úmidas de terra baixas crescem, em termos de biomassa/ha/ano, mais rapidamente do que as florestas semidecíduas e as montanas, as quais estão estabelecidas sob condições menos favoráveis ao crescimento vegetal. Todavia, as florestas de terras baixas apresentam menor resiliência (senso Begon et al., 1996) em comparação às outras, pois são mais desenvolvidas estruturalmente (biomassa, altura média, área basal) e, portanto, requerem maiores períodos de tempo para se regenerar.

Além de diferenças nos valores a serem restaurados, as florestas montanas apresentam características diferentes das florestas de terra baixas, que podem determinar padrões distintos de resiliência (Kappelle et al., 1996). Destaca-se a riqueza de árvores, o tamanho médio de diásporos e a importância relativa de grupos dispersores (Tabarelli, 1997). Tais características emergem como fatores controladores da velocidade de regeneração da floresta e podem explicar diferenças de resiliência entre as florestas tropicais.

No que se refere à mudança na composição de espécies durante a regeneração (sucessão senso Whitmore, 1991), a descrição de modelos sucessionais, como cronosseqüência de estabelecimento e extinção de espécies dominantes, tem permitido: (1) prever parte das modificações na composição florística e nas demais características da comunidade ao longo do processo de regeneração e (2) inferências sobre os mecanismos organizadores das transformações. Esse estudo propôs a investigação das seguintes questões: (1) quais as tendências de transformação das características da floresta, a ordem e a velocidade de restauração das mesmas após corte e queima? e (2) quais os modelos sucessionais dessa floresta montana?

\section{MATERIAL E MÉTODOS}

\section{Área de estudo}

O estudo foi desenvolvido no Núcleo Santa Virgínia $\left(45^{\circ} 30^{\prime}\right.$ a $45^{\circ} 11^{\prime} \mathrm{W}$ e $23^{\circ} 17^{\prime}$ a $\left.23^{\circ} 24^{\prime} \mathrm{S}\right)$, Parque Estadual da Serra do Mar, com área aproximada de 4.790 ha (Fig. 1). A área de estudo está situada na região de escarpas e reversos da Serra do Mar, no Planalto de Paraitinga-Paraibuna, entre altitudes que variam de $870 \mathrm{~m}$ a $1.100 \mathrm{~m}$. O relevo apresenta dissecação muito forte, com declividades variando entre $24^{\circ}$ e $37^{\circ}$ e dinâmica instável. Predominam na região solos dos tipos Latossolo Vermelho-Amarelo, Cambissolos e solos Litólicos (Radambrasil, 1983).

Conforme classificação climática de Koeppen, o clima regional é tropical temperado sem estação seca (Setzer, 1966). A precipitação média anual é de $2.180 \mathrm{~mm}$, sendo os meses mais úmidos: dezembro, janeiro e fevereiro; e os menos chuvosos: junho, julho e agosto. Não se observa nenhum mês com precipitação média inferior a $60 \mathrm{~mm}$ (São Paulo, 1972). De acordo com Veloso et al. (1991), a vegetação pode ser classificada como floresta ombrófila densa montana, uma das formações que compõe a floresta Atlântica brasileira.

Na década de 60, parte da floresta Atlântica no Núcleo Santa Virgínia sofreu corte raso e queima, sendo substituída por pastagens (Melinis minutiflora-Gramineae). Atualmente, apresentase na forma de mosaico, composto por áreas de floresta madura, pastagens, plantios de Eucalyptus spp. e florestas secundárias em diferentes idades de regeneração. As unidades que compõem esse mosaico representam um gradiente de regeneração entre a pastagem e a floresta madura. Informações detalhadas sobre a composição e a abundância de espécies arbóreo-arbustivas em florestas com diferentes idades no Núcleo Santa Virgínia podem ser obtidas em Tabarelli et al. (1993) e Tabarelli (1997). 


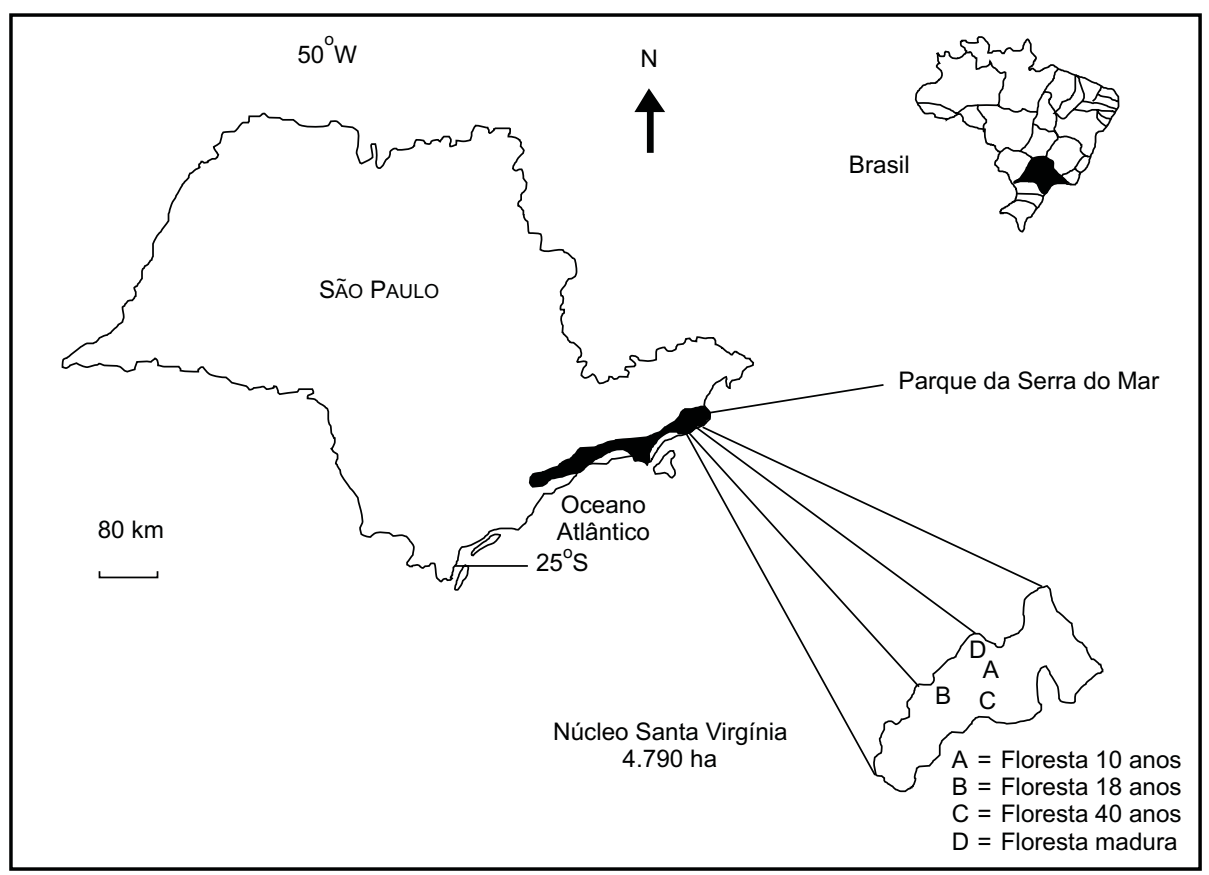

Fig. 1 - Mapa de localização do Núcleo Santa Virgínia, SP.

\section{Regeneração da floresta}

A análise das alterações nas características da floresta durante o processo de regeneração teve como base a descrição de trechos de florestas com diferentes idades. Foram selecionados três trechos de floresta secundária com, aproximadamente, 10, 18 e 40 anos de idade, estabelecidos na médiaencosta, onde a floresta madura foi cortada e queimada para o estabelecimento de pastagens.

Como controle foi selecionado um trecho de floresta madura sob a mesma condição topográfica, mas sem registro de perturbação antrópica severa. Em todos os trechos foram estabelecidas parcelas retangulares de $40 \times 50 \mathrm{~m}(0,2 \mathrm{ha})$, constituindo uma área amostral total de 0,8 ha. Em cada uma das parcelas foram amostrados todos os indivíduos com diâmetro do caule a altura do peito superior a $3,2 \mathrm{~cm}$.

Foram analisadas modificações em características da floresta relativas à: (1) estrutura física; (2) composição; (3) riqueza e diversidade; e (4) composição de guildas de espécies arbóreo-arbustivas.

As características de estrutura física foram: (1) densidade de indivíduos (número de indivíduos/ha); (2) área basal ( $\mathrm{m}^{2} / \mathrm{ha}$ ); e (3) volume de madeira (altura total $\mathrm{x}$ área basal, em $\mathrm{m}^{3} / \mathrm{ha}$ ). As modificações na composição de espécies foram analisadas no nível de família, enfatizando-se a importância relativa das principais famílias (riqueza na família/riqueza total x 100) nas florestas com diferentes idades, em relação à floresta madura. A importância relativa de Myrtaceae, Lauraceae, Melastomataceae e Rubiaceae nas diferentes florestas foi analisada através de teste $G$ (Sokal $\&$ Rohlf, 1995). As características de riqueza e de diversidade foram: (1) riqueza (número de espécies) e (2) diversidade de espécies, medida através dos índices de diversidade de Shannon-Wiener e de Simpson (Magurran, 1988).

A composição de guildas refere-se aos percentuais de espécies nas guildas de dispersão, estratificação e regeneração, sendo (1) guildas de dispersão: (a) espécies anemocóricas, aquelas que apresentam mecanismos que facilitam sua dispersão pelo vento, (b) zoocóricas, aquelas que apresentam características relacionadas à dispersão por animais e (c) autocóricas, espécies que dispersam por queda livre ou apresentam mecanismos de autodispersão (Pijl, 1982); (2) guildas de estratificação: (a) espécies de sub-bosque, aquelas que ocupam o estrato dos arbustos e das pequenas árvores no interior 
da floresta e (b) espécies de dossel, aquelas que ocupam o dossel da floresta e/ou o estrato emergente (Wilson, 1989); e (3) guildas de regeneração: (a) espécies intolerantes à sombra, aquelas que necessitam de clareiras como sítio de regeneração e (b) espécies tolerantes à sombra, aquelas capazes de se regenerar no sub-bosque da floresta. Nesse grupo encontram-se as espécies típicas de sub-bosque, as quais completam seus ciclos de vida no interior da floresta, e aquelas de dossel que toleram a sombra somente nos estádios nãoreprodutivos (Hartshorn, 1978).

Para a classificação das espécies nas diferentes guildas, foram utilizadas informações sobre suas biologias publicadas na Flora Ilustrada Catarinense (Reitz, 1965), em van Roosmalen (1985) e no conhecimento dos autores sobre as mesmas. Para analisar as relações entre as mudanças na composição de guildas e a regeneração da floresta, analisaramse as relações entre guildas e as características de estrutura física das florestas com 18, 40 anos e floresta madura, através do coeficiente de correlação de Pearson (Sokal \& Rohlf, 1995).

\section{Modelos de sucessã̃o}

Neste estudo, sucessão significa mudança direcional na composição de espécies (Begon et al., 1996), sendo modelos os conjuntos de estádios sucessionais, caracterizados pela ocorrência de espécies dominantes ao longo do processo de regeneração da floresta (Gómez-Pompa \& VázquezYanes, 1981). A descrição dos modelos de sucessão envolveu: (1) a análise dos quatro trechos de floresta amostrados; (2) a observação de áreas contíguas em estádio inicial de regeneração, como descritas em Tabarelli (1997); e (3) informações obtidas com moradores locais. Não foram estabelecidas réplicas das florestas devido à existência, na área de estudo, de apenas um trecho das florestas com 10 e com 40 anos.

\section{RESULTADOS}

\section{Regeneração da floresta}

As características de estrutura física da floresta transformam-se rapidamente em direção aos valores observados na floresta madura. A floresta com 40 anos apresentou $117,1 \%$ da densidade de indivíduos, $86,5 \%$ da área basal e $54,3 \%$ do volume observado na floresta madura (Tabela 1).

Nos quatro trechos de floresta foram amostradas 168 espécies arbóreo-arbustivas pertencentes a 43 famílias. Myrtaceae, Lauraceae, Melastomataceae e Rubiaceae estiveram entre as famílias com maiores riquezas de espécies, apresentando tendências de modificações em suas importâncias relativas durante a regeneração da floresta. Ocorreu aumento nas importâncias relativas de Myrtaceae e de Lauraceae e redução das de Melastomataceae e de Rubiaceae, durante a regeneração da floresta (Fig. 2), sendo estas diferenças significativas ( $G=$ $16,702 ;$ g.l. $=6 ; P=0,01)$. Na floresta com 40 anos, a importância relativa de Myrtaceae e Lauraceae correspondeu a $38,8 \%$ e $96,1 \%$, respectivamente, do valor de importância relativa destas famílias na floresta madura.

Ao longo do processo de regeneração da floresta ocorreu aumento na riqueza e na diversidade de espécies. A riqueza e a diversidade de espécies na floresta com 40 anos foram superiores às observadas na floresta madura, dependendo do índice utilizado (Tabela 2).

A velocidade com que a composição de guildas se transformou, em relação aos valores observados na floresta madura, dependeu da guilda analisada.

TABELA 1

Densidade de indivíduos, área basal e volume de madeira em quatro trechos de floresta Atlântica montana.

\begin{tabular}{|c|c|c|c|}
\hline Florestas & Densidade (ha) & Área basal (m²/ha) & Volume $\left(\mathrm{m}^{3} / \mathrm{ha}\right)$ \\
\hline 10 anos & 1.280 & 5,0 & 28,6 \\
\hline 18 anos & 3.325 & 23,4 & 226,9 \\
\hline 40 anos & 2.735 & 33,4 & 443,8 \\
\hline Madura & 2.335 & 38,6 & 818,0 \\
\hline
\end{tabular}


Importância relativa das famílias

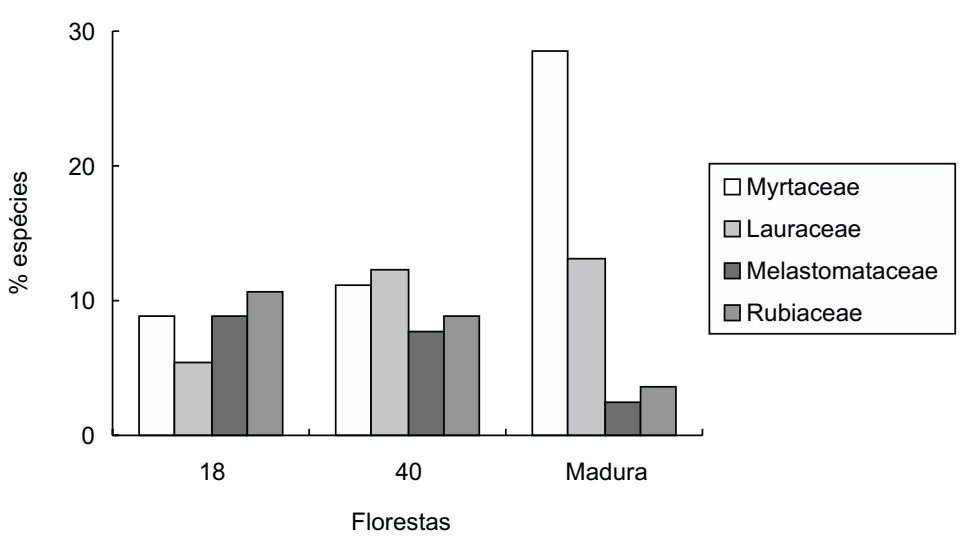

Fig. 2 - Importância relativa de Myrtaceae, Lauraceae, Melastomataceae e Rubiaceae em três trechos de floresta Atlântica montana com diferentes idades.

TABELA 2

Riqueza (S), índices diversidade de Shannon-Wiener (H') e de Simpson (1-D) em quatro trechos de floresta Atlântica montana.

\begin{tabular}{|l|l||c|c|}
\hline Florestas & $\mathbf{S}$ & H' (bits/ind.) & 1-D \\
\hline 10 anos & 4 & 1,161 \\
\hline 18 anos & 56 \\
\hline 40 anos & 90 \\
\hline Madura & 84 \\
\hline
\end{tabular}

Durante a regeneração da floresta ocorreu um aumento no percentual de espécies de subbosque, de espécies zoocóricas e daquelas tolerantes à sombra, havendo, conseqüentemente, uma redução daquelas de dossel, anemo/autocóricas e intolerantes à sombra (Tabela 3). A floresta com 40 anos apresentou $78,4 \%, 88,7 \%$ e $81 \%$ do percentual de espécies de sub-bosque, de espécies zoocóricas e daquelas tolerantes à sombra observado na floresta madura. Foram observadas relações entre a composição de guildas e as características de estrutura física das florestas com diferentes idades (Tabela 4), corroborando a hipótese de que a regeneração implica mudanças direcionais na composição de guildas de plantas arbustivo-arbóreas.

Conforme estes resultados, as características da floresta transformam-se em diferentes velocidades em direção aos valores observados na floresta madura. Em ordem decrescente de velocidade, a floresta, após corte e queima, restaura a riqueza e a diversidade de espécies, a composição de guildas, a composição florística (importância relativa das famílias) e, por último, os atributos da estrutura física, com exceção da densidade de indivíduos. A floresta com 40 anos apresentou entre $38,8 \%$ e $117,1 \%$ dos valores observados na floresta madura, dependendo da característica analisada.

\section{Modelos de sucessão}

A sucessão secundária da floresta montana, no local de estudo, pode ser caracterizada pela colonização e extinção de três ou quatro espécies pioneiras e pelo estabelecimento de uma espécie dominante tolerante à sombra (Fig. 3), dependendo do histórico de perturbação da área. Em locais onde não ocorreram queimadas, o processo de sucessão da floresta pode ser representado por quatro estádios sucessionais (Fig. 3, Modelo A).

Em locais onde o fogo foi utilizado com freqüência, observou-se a ocorrência de mais um estádio sucessional, dominado por Baccharis dracunculifolia (Compositae) (Fig. 3, Modelo B). 
TABELA 3

Percentual de espécies em diferentes guildas de estratificação: sub-bosque (SB), dossel (DS); dispersão: anemo/autocóricas (A/A), zoocóricas (ZO); e regeneração: tolerantes à sombra (TS) e intolerantes à sombra (IS), em quatro trechos de floresta Atlântica montana.

\begin{tabular}{|l|l|l|l|l|l|l|}
\hline Florestas & SB & DS & A/A & ZO & TS & IS \\
\hline 10 anos & 25,0 & 75,0 & 75,0 & 25,0 & 25,0 & 75,0 \\
\hline 18 anos & 41,0 & 58,9 & 21,4 & 78,5 & 55,3 & 44,6 \\
\hline 40 anos & 43,3 \\
\hline Madura & 55,2 & 56,6 & 12,2 & 87,7 \\
\hline 4 & 44,7 & 1,2 & 98,8 & 73,3 & 26,6 \\
\hline
\end{tabular}

TABELA 4

Correlação entre características de composição de guildas ( $\mathrm{SB}=\%$ espécies de sub-bosque, $\mathrm{ZO}=\%$ de zoocóricas, $\mathrm{TS}=\%$ de tolerantes à sombra) e de estrutura física da floresta Atlântica montana durante sua regeneração.

\begin{tabular}{|c|c|c|c|c|c|c|}
\hline Características & SB & ZO & TS & Densidade & Área basal & Volume \\
\hline SB & 1 & - & -- & - & - & - \\
\hline $\mathrm{ZO}$ & $0,963 *$ & 1 & - & - & - & - \\
\hline TS & 0,942 & 0,998 & 1 & - & - & - \\
\hline Densidade & $-0,905$ & $-0,987$ & $-0,950$ & 1 & - & - \\
\hline Área & 0,874 & 0,973 & 0,986 & $-0,998$ & 1 & - \\
\hline Volume & 0,985 & 0,995 & 0,986 & $-0,966$ & 0,945 & 1 \\
\hline
\end{tabular}

* $r=$ coeficiente de correlação de Pearson.

Estes modelos representam a substituição direcional de formas de crescimento e de histórias de vida, a partir de plantas herbáceas pioneiras até árvores tolerantes à sombra de ciclo de vida longo.

Entre as pioneiras, Pteridium aquilinum (Polypodiaceae) e Imperata brasiliensis (Gramineae) são herbáceas; Baccharis dracunculifolia e Tibouchina mutabilis (Melastomataceae) são árvores de ciclo de vida curto; e Alchornea triplinervia (Euphorbiaceae) é uma árvore pioneira de ciclo de vida longo. A ocorrência da floresta de Alchornea (80 anos) foi inferida a partir da abundância desta espécie na floresta com 40 anos e da informação de moradores locais.

\section{DISCUSSÃO}

\section{Regeneração da floresta}

De acordo com os resultados encontrados neste estudo, durante o processo de regeneração desta floresta montana, ocorre aumento da riqueza, da diversidade de espécies, do percentual de espécies zoocóricas, de sub-bosque, de espécies tolerantes à sombra e dos atributos físicos da floresta (área basal, volume), com exceção da densidade de indivíduos (Tabelas 1, 2 e 3 ).

Estas são tendências observadas durante o processo de regeneração da floresta Atlântica no Sul e Sudeste do Brasil (veja Klein, 1980; Mendonça et al., 1992; Leitão Filho, 1993; Feitosa do Nascimento, 1994; Torezan, 1995; Pessoa et al., 1997). Nesses estudos, durante a regeneração também se observaram aumentos na importância relativa das espécies de sub-bosque, das espécies zoocóricas e das tolerantes à sombra. A análise da composição de espécies dos trechos de floresta, descritos neste e no conjunto de estudos citados anteriormente, revela que estes processos refletem o desaparecimento de espécies pioneiras, como as dos gêneros Baccharis, Vernonia, Eupatorium (Compositae), Senna, Piptadenia, Dalbergia (Leguminosae) e Tibouchina, e o aparecimento de um maior número de espécies de Myrtaceae e Lauraceae, famílias compostas, em sua maioria, por espécies tolerantes à sombra, zoocóricas e, no caso de Myrtaceae, principalmente por espécies típicas de sub-bosque (Reitz, 1965). 


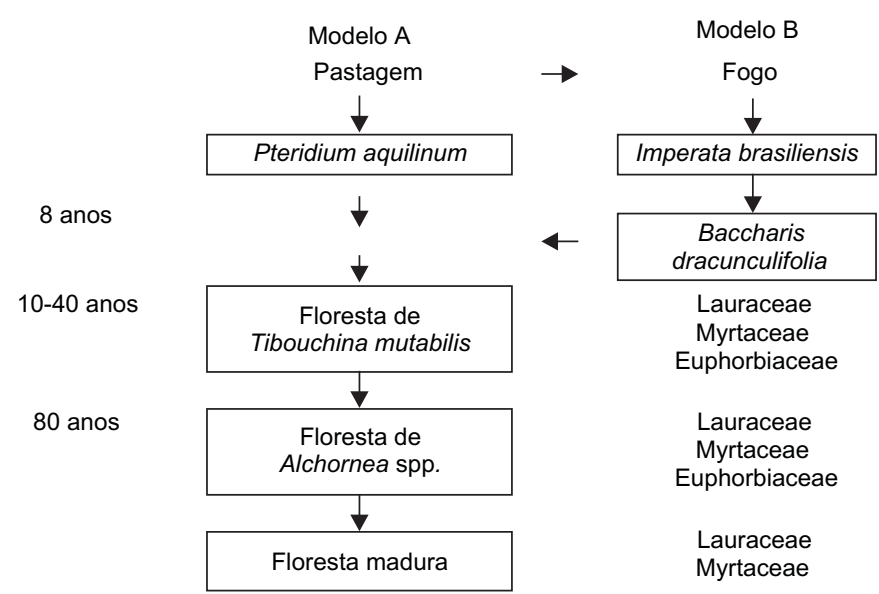

Fig. 3 - Modelos de sucessão secundária em uma floresta Atlântica montana após corte e queima.

Outra tendência observada refere-se à composição de arbustos e de pequenas árvores. Nas florestas em estádios iniciais de regeneração, as espécies de arbustos e pequenas árvores são pioneiras dos gêneros Miconia, Leandra (Melastomataceae), Piper (Piperaceae), Psychotria (Rubiaceae) e Rapanea (Myrsinaceae). Nas florestas mais velhas, são espécies tolerantes à sombra pertencentes à Myrtaceae e Monimiaceae, entre outras. Contudo, o processo responsável pela elevada riqueza e diversidade observadas em alguns trechos de florestas neotropicais em regeneração parece ser a ocorrência simultânea de espécies pioneiras e tolerantes à sombra (veja Laska, 1997).

No local de estudo, como em todas as florestas tropicais conhecidas, observa-se, durante o processo de regeneração, a redução da densidade total de indivíduos e o aumento da biomassa e do volume de madeira (Crow, 1980; Saldarriaga et al., 1988; Brown \& Lugo, 1990), conseqüência do crescimento dos indivíduos estabelecidos.

Conforme os resultados aqui encontrados, a floresta Atlântica montana, após perturbação antrópica, restaura, em ordem decrescente de velocidade, a riqueza e a diversidade de espécies, a composição de guildas, a composição florística e, por último, os atributos da estrutura física, com exceção da densidade de indivíduos. Estes resultados estão de acordo com aqueles obtidos em estudos sobre a regeneração de florestas tropicais que apresentam descrições completas de cronosseqüências ou com controle estabelecido (Uhl et al., 1982; Saldarriaga et al., 1988; Brown \& Lugo,
1990; Saldarriaga \& Uhl, 1991), com exceção da composição florística, para a qual não há informações disponíveis.

De acordo com Brown \& Lugo (1990) e Vandermeer et al. (1997), a riqueza e a diversidade de espécies transformam-se em velocidades distintas das quais se transformam as características de estrutura física da floresta. Independente do tipo de floresta, florestas secundárias com idades ao redor de 80 anos apresentam riquezas e diversidades de espécies similares às observadas nas florestas maduras, enquanto são necessários entre 100 e 200 anos para que as mesmas atinjam valores de biomassa similares aos da floresta madura.

Quanto ao tempo de regeneração das florestas tropicais, poucas generalizações e hipóteses são possíveis. Os resultados apresentados por Ewel (1980), Brown \& Lugo (1990), Saldarriaga \& Uhl (1991), Walschburger \& Von Hildebrand (1991), Sanchez \& Alvarez-Sanchez (1995) e Pessoa et al. (1997) permitem concluir que florestas com idades entre 60 e 80 anos apresentam características similares às observadas na floresta madura, com exceção de atributos de estrutura física, como a biomassa, confirmando as observações preliminares de Richards (1955) e de Budowski (1961).

Assumindo-se linearidade temporal na velocidade das modificações, os resultados deste estudo indicam que a floresta Atlântica montana regenera-se dentro dos padrões observados para o conjunto das florestas tropicais, já que a floresta com 40 anos apresentou entre $38,8 \%$ e $117,1 \%$ dos valores observados na floresta madura, dependendo 
do atributo analisado (Tabelas 1, 2 e 3). Este resultado está de acordo, também, com Klein (1980), o qual afirmou que florestas secundárias com idades entre 80 e 110 anos no baixo e médio Vale do Itajaí, em seus traços essenciais e sob todos os aspectos fisionômicos, muito se assemelhavam à floresta madura.

Uma das hipóteses possíveis é que, mantida a intensidade de perturbação constante, o tempo de regeneração das florestas tropicais constitui um intervalo no qual diferentes tipos florestais, como as florestas decíduas, perenifolias e montanas, estão situados. Conforme Ewel (1980), as florestas úmidas de terras baixas crescem mais rapidamente do que as florestas semidecíduas e as montanas, as quais estão estabelecidas sob condições menos favoráveis ao crescimento vegetal. Todavia, as florestas de terras baixas apresentariam menor resiliência (taxa de recomposição) em comparação às outras, pois são mais desenvolvidas em suas estruturas (biomassa, altura média) e, portanto, requerem maiores períodos de tempo para se regenerar. Estas florestas se situariam no extremo superior do intervalo de tempo.

Além dos aspectos da estrutura física, as florestas montanas apresentam características diferentes das florestas de terra baixas, que podem determinar diferentes padrões de resiliência. De modo geral, as florestas montanas, comparativamente às florestas neotropicais úmidas de terras baixas, apresentam: menor percentual de espécies com diásporos maiores do que $1,5 \mathrm{~cm}$ de comprimento; menor percentual de espécies dependentes de dispersores especializados; espécies pioneiras, preferencialmente anemocóricas; e menor riqueza de espécies, principalmente de árvores (Gentry, 1982; Gentry, 1988a; Tabarelli \& Mantovani, 1999).

Grande parte da riqueza da floresta Atlântica montana no Sudeste do Brasil deve-se a espécies com diásporos menores que $1,5 \mathrm{~cm}$ de comprimento, pertencentes a Myrtaceae, Melastomataceae, Rubiaceae, Monimiaceae, Flacourtiaceae, Myrsinaceae, Araliaceae, Olacaceae e as palmeiras do gênero Geonoma (Mantovani et al., 1990; Leitão Filho, 1993). São espécies dispersas, preferencialmente, por passeriformes, pequenos frugívoros generalistas da família Pipridae e subfamília Thraupinae (Stiles \& Rosselli, 1993; Sick, 1997), espécies do gênero Turdus (Turdinae) e, no caso de Myrtaceae, também por frugívoros de médio e grande portes (Moraes, 1992; Sick, 1997).
Não são comuns nesta floresta espécies com grandes sementes, como as amazônicas Bertholletia excelsa (Lecythidaceae), Vouacapoua americana (Leguminosae) e Astrocaryum paramaca (Palmae), dispersas por roedores caviomorfos a curtas distâncias (Mori \& Prance, 1990; Forget, 1990, 1992), e que necessitam ser enterradas para obterem sucesso na germinação (Forget, 1992). Também não são comuns espécies com médias $\mathrm{e}$ grandes sementes dispersas por morcegos, como algumas dos gêneros Lecythis e Eschweilera (Lecythidaceae) (Mori, 1989). Em quatro trechos de floresta de terra firme na Amazônia brasileira, Almeida et al. (1993) amostraram entre cinco e nove espécies de Eschweilera por hectare. Além destas, as florestas úmidas de terras baixas na Amazônia são ricas em espécies de Sterculiaceae, Sapotaceae, Palmae e Leguminosae (Gentry, 1982; Gentry, 1990), famílias com elevado número de espécies com grandes sementes (van Roosmalen, 1985).

Na floresta Atlântica montana, os estádios arbustivo-arbóreos pioneiros são dominados, em termos de número de indivíduos, por espécies anemocóricas, como aquelas dos gêneros Baccharis e Tibouchina (Klein, 1980). Este não é o padrão observado em outras florestas tropicais. Em florestas úmidas de terra firme na Amazônia, se não a maior parte das árvores pioneiras, pelo menos as mais abundantes são zoocóricas, como aquelas dos gêneros Cecropia (Cecropiaceae), Vismia (Guttiferae) e Miconia (Gómez-Pompa et al., 1991; Vieira et al., 1996).

Em relação à riqueza, florestas neotropicais de terras baixas apresentam entre 118 e 307 espécies de árvores $(\oplus 10 \mathrm{~cm}$ dap) por hectare (Gentry, 1988b; Valencia et al., 1994). No local de estudo foram amostradas 136 espécies de árvores em 1 ha (Tabarelli \& Mantovani, 1999). Estas características de riqueza e de composição de guildas podem afetar a velocidade de chegada e a probabilidade de estabelecimento das espécies, o número de estádios sucessionais e os valores de riqueza a serem restaurados, conferindo maior resiliência à floresta Atlântica montana.

O padrão encontrado no local de estudo é um dos possíveis padrões de regeneração da floresta Atlântica montana, considerando-se todas as variáveis que podem afetar a velocidade de regeneração das florestas tropicais. Características desta floresta, como a riqueza de árvores e o tamanho 
médio dos diásporos, sugerem que, mantidas a mesma intensidade de perturbação e a matriz florestal circundante, esta floresta apresenta maior resiliência do que as florestas neotropicais úmidas de terras baixas. Características diversas, como a distribuição de diásporos por classes de tamanho e a importância relativa de grupos dispersores, podem constituir fatores controladores da velocidade de regeneração.

Do ponto de vista aplicado, os resultados deste estudo sugerem que a importância relativa de Myrtaceae e de Lauraceae pode constituir um indicador do estádio de regeneração ou de degradação desta floresta, não só relativo à riqueza, mas também à disponibilidade de recursos e à composição de guildas. Espécies destas famílias representam grande parte dos recursos para frugívoros de médio e grande porte (veja Moraes, 1992; Petroni, 1993; Sick, 1997) e, no caso de Myrtaceae, representa grande parte das árvores e arvoretas típicas de sub-bosque que compõem a floresta madura (Reitz, 1965).

\section{Modelos de sucessã̃o}

No local de estudo, a sucessão pode ser dividida em quatro ou cinco estádios sucessionais, dependendo da intensidade da perturbação. Caracteriza-se não só pela substituição de espécies intolerantes à sombra por tolerantes, mas também pela substituição direcional de formas de crescimento e de histórias de vida a partir de espécies herbáceas, dominando, consecutivamente, arbustos, árvores de ciclo de vida curto e de ciclo de vida longo (Fig. 3).

A sucessão secundária, conforme os modelos descritos neste e em outros estudos (Klein, 1980; Gómez-Pompa \& Vázquez-Yanes, 1981; Uhl, 1987; Whitmore, 1990), apresenta várias similaridades nos diferentes tipos de florestas tropicais. A primeira delas refere-se ao fato de que a cronosseqüência de substituição das espécies dominantes representa a substituição direcional de formas de crescimento e de histórias de vida. Após perturbação antrópica, na maioria dos casos corte e queima da floresta e uso agropastoril, observa-se o estabelecimento e a extinção de ervas, arbustos, árvores pioneiras de ciclo de vida curto e árvores pioneiras de ciclo de vida longo, consecutivamente.

Como exemplo, a sucessão de uma floresta tropical no México pode ser dividida em cinco estádios sucessionais: o primeiro caracterizado pelo estabelecimento de ervas, o segundo, por arbustos (Piper-Piperaceae, Solanum-Solanaceae e Urera-Urticaceae), o terceiro, por árvores pioneiras pequenas (Heliocarpus-Sterculiaceae e Trema-Ulmaceae), o quarto, por árvores pioneiras grandes (Cecropia e Didymopanax-Araliaceae) e o quinto, pelo estabelecimento de espécies da floresta madura (Gómez-Pompa \& Vázquez-Yanes, 1981).

Esta similaridade de organização ocorre, também, em nível taxonômico, no qual grupos de espécies relacionadas taxonomicamente ocupam as mesmas posições dentro da cronosseqüência, em toda a região tropical. É o caso das espécies de Imperata, Gleichenia (Gleicheniaceae) e de Pteridium aquilinum, táxons herbáceos que ocorrem em estádios iniciais da sucessão, em florestas neo e paleotropicais (Klein, 1980; Woods, 1989; Riswan \& Kartawinata, 1991; Nykvist, 1996; Turner et al., 1996).

Na floresta Atlântica no Sul e Sudeste do Brasil, a sucessão apresenta similaridades florísticas marcantes em nível genérico. Como no local de estudo, as seqüências de substituição: BaccharisTibouchina-Alchornea ou Baccharis-RapaneaTibouchina-Alchornea são de ampla ocorrência nos domínios dessa floresta (Klein, 1980; Tabarelli, 1992; Feitosa do Nascimento, 1994; Pessoa et al. 1997). A similaridade florística não se restringe às espécies dominantes, estendendo-se às demais espécies pioneiras que ocorrem nas florestas em regeneração. As espécies pioneiras que ocorrem ao longo da encosta Atlântica são de ampla distribuição, ressaltando-se as dos gêneros: Piptocarpha e Vernonia (Compositae), Cecropia, Clethra (Clethraceae), Alchornea, Hyeronima, Pera (Euphorbiaceae), Senna, Miconia, Tibouchina, Rapanea (Myrsinaceae) e Solanum, como ressaltado por Mantovani (1993).

A similaridade não se restringe ao grupo de espécies pioneiras, mas abrange as espécies tolerantes à sombra. São comuns nestas florestas: Casearia sylvestris, C. decandra, C. obliqua (Flacourtiaceae), Sloanea monosperma, S. guianensis (Elaeocarpaceae), Endlicheria paniculata, Nectandra oppositifolia, Ocotea puberula (Lauraceae), Guarea macrophylla (Meliaceae), Mollinedia elegans, M. schottiana (Monimiaceae), Guapira opposita (Nyctaginaceae), Posoqueria acutifolia, Psychotria cartaginensis, P. leiocarpha, P. suterella (Rubiaceae), Allophyllus edulis, 
Cupania vernalis e Matayba guianensis (Sapindaceae), as quais ocorrem até o limite sul desta floresta, no Estado Rio Grande do Sul (veja Tabarelli, 1992; Sevegnani, 1995).

A segunda similaridade observada no processo de sucessão refere-se à relação existente entre o número de estádios sucessionais e a intensidade de perturbação da floresta. No local de estudo, em áreas ocupadas com cultivo de subsistência e submetidas ao fogo, a regeneração da floresta inclui o estabelecimento de um estádio dominado por Baccharis, anterior ao estabelecimento de Tibouchina (Fig. 3). Em áreas não submetidas ao fogo, não se observa este estádio sucessional. Em outros locais na floresta Atlântica, o uso intensivo da área está associado ao aparecimento de estádios dominados por Baccharis e por Rapanea, o que Klein (1980) denominou de capoeirinha e capoeira. A ocorrência e/ou a dominância de espécies de Imperata e Pteridium aquilinum, por longos períodos, tem sido considerada como indicador de áreas submetidas a perturbação intensa, onde a regeneração da floresta tende a ocorrer de forma lenta (veja Whitmore, 1991; Fox et al., 1997).

Conforme Woods (1989), Riswan \& Kartawinata (1991) e Nykvist (1996), o uso intensivo das áreas degrada as condições físicas e químicas do solo, o que confere maior habilidade competitiva às plantas herbáceas e arbustivas, comparativamente às arbóreas (Kellman, 1980). Além disso, o uso intensivo da área elimina possíveis fontes de regeneração para as espécies tolerantes à sombra, como diásporos e rebrotas (Uhl et al., 1982; Guariguata \& Dupuy, 1997). A degradação das condições químicas e físicas do solo, conseqüência do uso intensivo da área, determina um aumento no número de estádios sucessionais ou a estabilização do processo de regeneração (Uhl et al., 1982; Whitmore, 1990, 1991; Walker et al., 1996). Os modelos sucessionais observados neste estudo poderiam ser definidos como de sucessão obrigatória (Horn, 1976), o qual pressupõe o estabelecimento de uma comunidade pioneira como um pré-requisito para o estabelecimento seqüencial de outros grupos de espécies. A ocorrência inicial isolada de espécies pioneiras e a relação observada entre a intensidade da perturbação e o número de estádios sucessionais sugerem que o estabelecimento da comunidade colonizadora não é um processo acidental.
Conforme Uhl (1987), a sucessão da floresta tropical após corte e queima pode ser melhor descrita pelo modelo de facilitação (Connell \& Slatyer, 1977). As espécies invadem lentamente um sítio disponível à colonização e facilitam o estabelecimento de outras espécies, pois agem como abrigo para os vetores de dispersão, melhoram as condições de fertilidade do solo e fornecem hábitats adequados ao recrutamento.

Ao contrário, Woods (1989) concluiu que a regeneração da floresta depende da capacidade das árvores pioneiras de vencerem a competição com as espécies herbáceas.

Essas afirmações corroboram a hipótese de Finegan (1984), para o qual um conjunto de processos está envolvido nas transformações florísticas observadas durante o processo de regeneração da floresta.

Agradecimentos - A Fundação de Amparo à Pesquisa do Estado de São Paulo pela Bolsa de Doutorado concedida (Processo no 93/3717-8).

\section{REFERÊNCIAS BIBLIOGRÁFICAS}

ALMEIDA, S. S., LISBOA, P. S. \& SILVA, A. S. L., 1993, Diversidade florística de uma comunidade arbórea na Estação Científica "Ferreira Penna", em Caxiuanã (Pará). Bol. Mus. Para. Emilio Goeldi, 9: 93-128.

BEGON, M., HARPER, J. L. \& TOWNSEND, C. R., 1996, Ecology: individuals, populations and communities. Blackwell, Oxford.

BROWN, S. \& LUGO, A. E., 1990, Tropical secondary forests. J. Trop. Ecol., 6: 1-32.

BUDOWSKI, G., 1961, Studies on forest succession in Costa Rica and Panama. P.h.D. Dissertation, Yale University, New Haven.

BUDOWSKI, G., 1965, Distribution of tropical American rain forest species in the light of successional process. Turrialba, 15: 40-2.

CONNELL, J. H. \& SLATYER, R. O., 1977, Mechanisms of succession in natural communities and their role in community stability and organization. Am. Nat., 111: 1119-1140.

CROW, T. R., 1980, A rainforest chronicle: a 30-year record of change in structure and composition at El Verde, Puerto Rico. Biotropica, 12: 42-55.

EWEL, J., 1980, Tropical succession: manifold routes to maturity. Biotropica, 12: 2-7.

FEITOSA DO NASCIMENTO, F. A., 1994, A sucessão secundária inicial na mata atlântica, sobre a Serra de Paranapiacaba, Ribeirão Grande, SP. Dissertação de Mestrado, Instituto de Biociências, Universidade de São Paulo, São Paulo. 
FINEGAN, B., 1984, Succession. Nature, 312: 109-115.

FORGET, P., 1990, Seed dispersal of Vouacapoua americana Aublet (Caesalpinaceae) by caviomorph rodents. J. Trop. Ecol., 6: 459-468.

FORGET, P., 1992, Seed removal and seed fate in Gustavia superba (Lecythidaceae). Biotropica, 24: 408-414.

FOX, B. J., TAYLOR, J. F., FOX, M. D. \& WILLIAMS, C., 1997, Vegetation changes across edges of rainforest remnants. Biol. Conserv., 82: 1-13.

GENTRY, A. H., 1982, Patterns of neotropical plant species diversity. Evol. Biol., 15: 1-84.

GENTRY, A. H., 1988a, Changes in plant community diversity and floristic composition on environmental and geographical gradients. Ann. Missouri Bot. Gard., 75: 1-34.

GENTRY, A. H., 1988b, Tree species richness of upper Amazonian forests. Proc. Natl. Acad. Sci., 85: 156-159.

GENTRY, A. H., 1990, Floristic similarities and differences between Southern Central America and upper and Central Amazonia, pp. 141-160. In: A. H. Gentry (ed.), Four neotropical rain forests, Yale University Press, London.

GÓMEZ-POMPA, A.G. \& VÁZQUEZ-YANES, C. N., 1981, Successional studies of a rain forest in Mexico, pp. 247 266. In: D. C. West, H. H. Schugart \& D. B. Botkin (eds.), Forest concepts and application, Springer-Verlag, New York.

GÓMEZ-POMPA, A., WHITMORE, T. C. \& HADLEY, M., 1991, Tropical rain forest: regeneration and management. Blackwell, New York.

GUARIGUATA, M. R. \& DUPUY, J. M., 1997, Forest regeneration in abandoned logging roads in lowland Costa Rica. Biotropica, 29: 15-28.

HARTSHORN, G. S., 1978, Treefalls and tropical forest dynamics, pp. 617-638. In: P. B. Tomlinson \& H. H. Zimmermann (eds.), Tropical trees as living systems, Cambridge Univ. Press, New York.

HORN, H. S., 1976, Succession, pp. 187-204. In: R. M. May (ed.), Theorical ecology, Blackwell, Oxford.

KAPPELLE, M., GEUZE, T., LEAL, M. \& CLEF, M., 1996, Successional age and forest structure in a Costa Rica upper montane Quercus forest. J. Trop. Ecol., 12: 681698.

KELLMAN, M., 1980, Geographic patterning in tropical weed communities and early secondary successions. Biotropica, 12 (supplement 1): 34-39.

KLEIN, R. M., 1980, Ecologia da flora e vegetação do Vale do Itajaí. Sellowia, 32: 165-389.

LASKA, M. S., 1997, Structure of understory shrub assembleges in a adjacent secondary and old growth tropical wet forests, Costa Rica. Biotropica, 29: 29-37.

LEITÃO FILHO, H. F. (org.), 1993, Ecologia da Mata Atlântica em Cubatão. Editora da UNESP, Editora da Universidade de Campinas, Campinas.

MAGURRAN, A. E., 1988, Ecological diversity and its measurement. Croom Helm, London.
MANTOVANI, W., 1993, Estrutura e dinâmica da floresta Atlântica na Juréia, Iguape, SP. Tese de Livre-docência, Instituto de Biociências, Universidade de São Paulo, São Paulo.

MANTOVANI, W., RODRIGUES, R. R., ROSSI, L., ROMANIUC-NETO, S., CATHARINO, E. L. M. \& CORDEIRO, I., 1990, A vegetação na Serra do Mar em Salesópolis. In: Simpósio de Ecossistemas da Costa Sul e Sudeste: estrutura, composição e manejo, Academia de Ciências do Estado de São Paulo, Anais, 2: 348-384.

MENDONÇA, R. R., POMPÉIA, S. L. \& MARTINS, S. E., 1992, A sucessão secundária da mata atlântica na Região de Cubatão. In: Instituto Florestal (ed.), II Congresso Nacional sobre Essências Nativas, São Paulo, Anais, 1: 131138.

MORAES, P. L. R., 1992, Dispersão de sementes pelo mono carvoeiro (Brachyteles arachnoides Geoffroy, E.1806) no Parque Estadual de Carlos Botelho. In: II Congresso Nacional sobre Essências Nativas, São Paulo, Anais, 4: 1199-1205.

MORI, S. A., 1989, Diversity of Lecythidaceae in the Guianas, pp. 319-322. In: L. B. Holm-Nielsen, I. C. Nielsen \& H. Balslev (eds.), Tropical forest, Academic Press, New York.

MORI, S. A. \& PRANCE, G. T., 1990, Taxonomy, ecology and economic botany of the Brazil nut (Bertholletia excelsa Humb. and Bonpl.: Lecythidaceae). Adv. Econ. Bot., 8: 130-150.

NYKVIST, N., 1996, Regrowth of secondary vegetation after the "Borneo fire" of 1982-1983. J. Trop., 12: 307-312.

PESSOA, S. V. A., GUEDES-BRUNI, R. R. \& BRUNO, C. K., 1997, Composição florística e estrutura do componente arbustivo-arbóreo de um trecho secundário de floresta montana na Reserva Ecológica de Macaé de Cima, pp. 147-168. In: H. C. de Lima \& R. R. GuedesBruni (eds.), Serra de Macaé de Cima: diversidade florística e conservação em Mata Atlântica, Jardim Botânico do Rio de Janeiro, Rio de Janeiro.

PETRONI, L. M., 1993, Aspectos da ecologia e comportamento do monocarvoeiro, Brachyteles sarachnoides (E. Geoffroy, 1806), (Cebidae-Primates), na Fazenda Intervales, Serra de Paranabiacaba, SP. Dissertação de Mestrado, Pontifícia Universidade Católica, Porto Alegre.

PIJL, VAN DER., 1982, Principles of dispersal in higher plants. $3^{a}$ ed., Springer-Verlag, Berlin.

RADAMBRASIL, 1983, Projeto Radambrasil: levantamento de recursos naturais. IBGE, Rio de Janeiro.

REITZ, R., 1965, Flora ilustrada catarinense. Herbário Barbosa Rodrigues, Itajaí, SC.

RISWAN, S. \& KARTAWINATA, K., 1991, Regeneration after disturbance in lowland mixed Dipterocarpaceae forest in East Kalimantan, pp. 295-301. In: A. Gómez-Pompa, T. C. Whitmore \& M. Hadley (eds.), Tropical rain forest: regeneration and management. Blackwell, New York.

RICHARDS, P. W., 1955, The secondary succession in the tropical rain forest. Sci. Prog. London, 43: 45-57. 
SALDARRIAGA, J. G., WEST, D. C., THARP, M. L. \& UHL, C., 1988, Long-term chronosequence in the upper Rio Negro of Colombia and Venezuela. J. Ecol., 76: 938958.

SALDARRIAGA, J. G. \& UHL, C., 1991, Recovery of forest vegetation following slash-and-burn agriculture in the upper rio Negro, pp. 303-312. In: A. Gomez-Pompa, T. C. Whitmore \& M. Hadley (eds.), Tropical rain forest: regeneration and management, Blackwell, New York.

SANCHEZ, G. \& ALVAREZ-SANCHEZ, J., 1995, Litterfall in primary and secondary tropical forests of Mexico. Trop. Ecol., 36: 191-201.

SÃo PAULO, 1972, Atlas pluviométrico do Estado de São Paulo. Secretaria de Serviços e Obras Públicas, São Paulo.

SETZER, J., 1966, Atlas climático e ecológico do Estado de São Paulo. Comissão Interestadual da Bacia ParanáUruguai, São Paulo.

SEVEGNANI, L., 1995, Fitossociologia de uma floresta secundária, Maquiné, RS. Dissertação de Mestrado, Instituto de Biociências, Universidade Federal do Rio Grande Sul.

SICK, H., 1997, Orinitologia brasileira. Editora Nova Fronteira, Rio de Janeiro.

SOKAL, R. R \& ROHLF, F. G., 1995, Biometry. W. H. Freeman and Company, New York.

STILES, F. G. \& ROSSELI, L., 1993, Consumption of fruits of the Melastomataceae: how diffuse is coevolution. Vegetatio, 107/108: 57-73.

TABARELLI, M., 1992, Flora arbórea da floresta estacional baixo-montana no Município de Santa Maria, RS, Brasil. In: II Congresso Nacional sobre Essências Nativas, São Paulo, Ver. Inst. Ftal., 4: 260-268.

TABARELLI, M., 1997, A regeneração da floresta Atlântica montana. Tese de Doutorado, Instituto de Biociências, Universidade de São Paulo, São Paulo.

TABARELLI, M. \& MANTOVANI, W., 1999, A riqueza da floresta Atlântica de encosta no Estado de São Paulo (Brasil). Rev. Brasil. Bot. (no prelo).

TABARELli, M., VILlANI, J. P. \& MANTOVANI, W., 1993, Aspectos da sucessão secundária no Parque Estadual da Serra do Mar, SP. Revta Inst. Ftal., 5: 101-114.

TOREZAN, J. M. D., 1995, Estudo da sucessão secundária, na floresta ombrófila densa submontana, em áreas anteriormente cultivadas pelo sistema de "coivara", em Iporanga, SP. Dissertação de Mestrado, Setor de Ciências Agrárias, Universidade Federal do Paraná.

TURNER, I. M., TAN, H. T. W. \& CHUA, K. S., 1996, Relationships between herb layer and canopy composition in tropical rain forest successional mosaic in Singapore. J. Trop. Ecol., 12: 843-851.

UHL, C., 1987, Factors controlling succession following slash-and-burn agriculture in Amazonia. J. Ecol., 75: 377-407.
UHL, C., JORDAN, C., CLARK, K., CLARK, H. \& HERRERA, R., 1982, Ecosystem recovery in Amazon caatinga forest after cutting, cutting and burning, and bulldozer clearing treatments. Oikos, 38: 313-320.

UNESCO/PNUMA/FAO, 1980, Ecosistemas de los bosques tropicales. UNESCO/CIFA, Paris.

VALENCIA, R., BALSLEV, H. \& PAZ Y MIÑO, G., 1994, High tree alpha-diversity in Amazonian Ecuador. Biodiver. Conser., 3: 21-28.

VANDERMEER, J., de la CERDA, I. G. \& BOUCHER, D., 1997, Contrasting growth rate patterns in eighteen tree species from a post-hurricane forest in Nicaragua. Biotropica, 29: 151-161.

VAN ROOSMALEN, G. M., 1985, Fruits of the Guianan flora. Institute of Systematic Botany, Utrecht University, Holanda.

VELOSO, H. P., RANGEL-FILHO, A. L. R. \& LIMA, J. C. A., 1991, Classificação da vegetação brasileira adaptada a um sistema universal. IBGE, Rio de Janeiro.

VIEIRA, I. C. G., SALOMÃO, R. P., ROSA, N. A., NEPSTAD, D. C. \& ROMA, J. C., 1996, O renascimento da floresta no rastro da agricultura. Ciên. Hoje, 20: 38 44.

WALKER, L. R., ZARIN, D. J., FETCHER, N., MYSTER, R. W. \& JOHNSON, A. H., 1996, Ecosystem development and plant succession on landslides in the Caribbean. Biotropica, 28: 566-576.

WALSCHBURGER, T. \& VON HILDEBRAND, P., 1991 The first 26 years of forest regeneration in natural and man-made gaps in the Colombian Amazon, pp. 257-263. In: A. Gómez-Pompa, T. C. Whitmore \& M. Hadley (eds.), Tropical rain forest: regeneration and management, Blackwell, New York.

WHITMORE, T. C., 1990, An introduction to tropical rain forests. Blackwell, London.

WHITMORE, T. C., 1991, Tropical rain forest dynamics and its implications for management, pp. 67-89. In: A. Gómez-Pompa, T. C. Whitmore \& M. Hadley (eds.), Tropical rain forest: regeneration and management, Blackwell, New York.

WILSON, J. B., 1989, A null model of guild proportionality, applied to stratification of a New Zealand temperate rain forest. Oecologia, 80: 263-267.

WOODS, P., 1989, Effects of logging, drought, and fire on structure and composition of tropical forests in Sabah, Malaysia. Biotropica, 21: 290-298. 